\begin{abstract}
A. Kravchuk ${ }^{\mathrm{a}}$, Z. Rymuza ${ }^{\mathrm{b}}$
${ }^{a}$ Belarussian National Technical University, Department of Theoretical Mechanics, Minsk, Belarus

${ }^{\mathrm{b}}$ Warsaw University of Technology, Institute of Micromechanics and Photonics, Warsaw, Poland
\end{abstract}

\title{
Model of a superlattice indentation
}

This paper deals with the problem of penetration of a conical indenter into a multilayer rigid-perfectly-plastic body. The penetration of the indenter is studied on the basis of Haar and von Karman hypothesis. The analytical distribution of the contact stress is obtained. A relationship between Meyer hardness and yield stresses of the layers is established.

Keywords: Rigid-perfectly-plastic body; Superlattice (multilayer) body; Hardness; Conical indenter

\section{Introduction}

Many numerical investigations of the plastic flow of the homogeneous rigid-perfectly-plastic bodies have been made $[1,2]$. The basic equations of axially symmetric plastic fields are well known. It was shown that these equations are statically determined when the Haar and von Karman hypothesis is satisfied [1, 2]. But there are no analytical investigations on the contact problem of a rigid indenter and a multilayer rigid-perfectly-plastic body. Its solution can be used as a model of tests on the hardness of composite materials.

\section{Basic suppositions}

The thickness $d_{j}$ and the yield stress $\sigma_{s, j}$ of a layer with number $j$ are a constant value. The conical indenter interact with $N$ layers (Fig. 1). The layers are made from rigid-perfectly-plastic materials. Haar and von Karman hypothesis is satisfied for each layer separately $[1,2]$ :

$\sigma_{1, j}(r, z)=\sigma_{3, j}(r, z)-2 \cdot K_{j}, \quad \sigma_{2, j}(r, z)=\sigma_{3, j}(r, z)$

where $\sigma_{i, j}(r, z)$ is a component of principal stress for layer with number $j(j=1, \cdot s, N), K_{j}=\sigma_{s, j} / 2$.

The contact problem can be conveniently studied with the help of cylindrical polar co-ordinates $(r, \theta, z)$, where $z$ is the axis of symmetry of the bodies (Fig. 1). The surface of the half-space at the $z-r$ plain after indenter penetration is defined by the equation [3]:

$z=\tan (\beta) \cdot r+\Delta$

where $\beta$ is a negative angle between the element of a cone and the $r$-direction (Fig. 1). The depth of penetration $\Delta$ is defined by the equation (Fig. 1): $\Delta=-\tan (\beta) \cdot a$, where $a$ is the radius of the contact area.

Let us consider a set of points $\left\{a_{j}\right\}_{j=0}^{N}$, where $a_{0}=a$, $a_{j, j=\overline{1, \ldots,(N-1)}}=a+\tan (\beta) \cdot\left(\sum_{i=1}^{j} d_{i}\right), a_{N}=0$. The segment $\left(a_{1}, a_{0}\right)$ is the contact area of the first layer with the conical

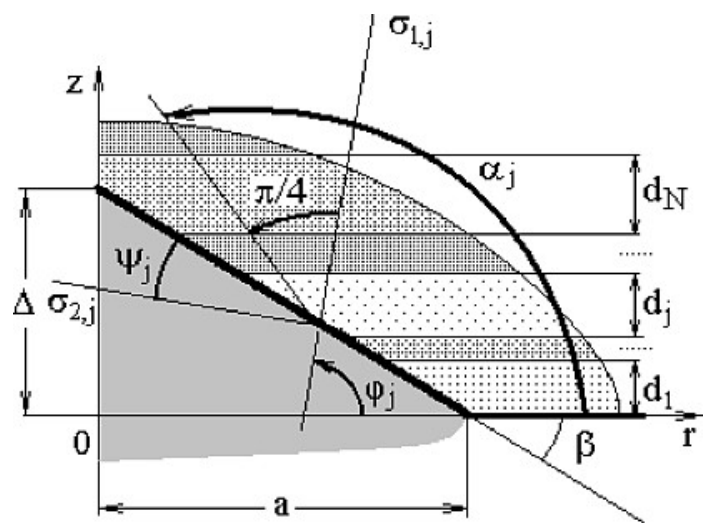

Fig. 1. Plane section of the contact of an axially symmetric indenter. $\varphi_{j}(r, z)$ is the angle between the $r$-direction and the first principle stress, $\psi_{j}(j=\overline{1, \ldots, N})$ is the constant angle which is defined by the direction of plastic shear in the contact area for layer with number $j, d_{\mathrm{N}}$, $d_{\mathrm{j}}, d_{1}$ are the thickness of the $N$-th, $j$-th and first layer, respectively, $\alpha_{1}=\frac{\pi}{4}+\varphi_{j}, \beta$ is the negative angle between the element of a cone and the $r$-direction, $a$ is the radius of the contact area, $\Delta=-\tan (\beta) \cdot a$ in the depth of the indentation, $\sigma_{1, j}$ and $\sigma_{2, j}$ are the directions of the principal stress for layer with number $j(j=1, \ldots, N)$.

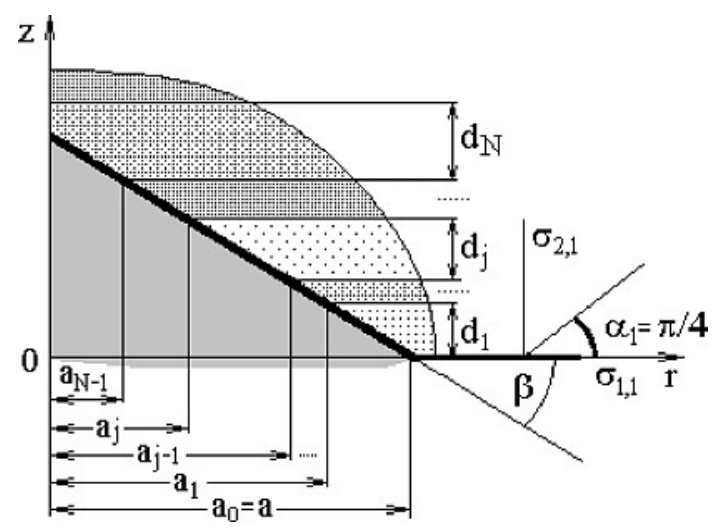

Fig. 2. Set of nodes $\left\{a_{j}\right\}_{j=0}^{N}$ and orientation of the principal stress on the free boundary.

indenter. Further, the segment $\left(a_{j}, a_{j-1}\right]$ is the contact area of the $j$-th layer $(j=2, \ldots, N)$ with the conical indenter (Fig. 2).

\section{Stress-state of a layer with number $j$}

Let us use the conditions of "full plasticity" for a layer in the form $[1,2]$ :

$\sigma_{1, j}(r, z)=\sigma_{3, j}(r, z)-2 \cdot K_{j}, \quad \sigma_{2, j}(r, z)=\sigma_{3, j}(r, z)$

where $\sigma_{i, j}(r, z)$ is a component of principal stress for layer with number $j(j=1, \ldots, N), K_{j}=\sigma_{s, j} / 2$. The stress-state 
in the layer with number $j$ involves the four stress components $\sigma_{r, j}(r, z), \sigma_{\theta, j}(r, z), \sigma_{z, j}(r, z), \tau_{r z, j}(r, z)$. The circumferential stress $\sigma_{\theta, j}(r, z)$ is a principal stress. The following equations are valid $[1,2]$ (Fig. 1):

$\sigma_{r, j}(r, z)=\sigma_{j}(r, z)-K_{j} \cdot \sin \left(2 \cdot \alpha_{j}(r, z)\right)$

$\sigma_{z, j}(r, z)=\sigma_{j}(r, z)+K_{j} \cdot \sin \left(2 \cdot \alpha_{j}(r, z)\right)$

$\sigma_{\theta, j}(r, z)=\sigma_{j}(r, z)+K_{j}$

$\tau_{r z, j}(r, z)=K_{j} \cdot \cos \left(2 \cdot \alpha_{j}(r, z)\right)$

where

$\sigma_{j}(r, z)=\frac{1}{2}\left(\sigma_{1, j}(r, z)+\sigma_{2, j}(r, z)\right), \alpha_{j}(r, z)=\varphi_{j}(r, z)+\pi / 4$

$\varphi_{j}(r, z)$ is angle between the $r$-direction and the first principle stress (Fig. 1).

These components satisfy the equation of equilibrium $[1,2]$ :

$\frac{\partial \sigma_{r, j}(r, z)}{\partial r}+\frac{\partial \tau_{r z, j}(r, z)}{\partial z}+\frac{\sigma_{r, j}(r, z)-\sigma_{\theta, j}(r, z)}{r}=0$

$\frac{\partial \tau_{r z, j}(r, z)}{\partial r}+\frac{\partial \sigma_{z, j}(r, z)}{\partial z}+\frac{\tau_{r z, j}(r, z)}{r}=0$

We substitute Eq. (2) into Eq. (3) and obtain the following system for each separate $j$-th layer:

$$
\begin{aligned}
& \frac{\partial \sigma_{j}(r, z)}{\partial r}-2 \cdot K_{j} \cdot \cos \left(2 \cdot \alpha_{j}(r, z)\right) \\
& \times \frac{\partial \alpha_{j}(r, z)}{\partial r}-2 \cdot K_{j} \cdot \sin \left(2 \cdot \alpha_{j}(r, z)\right) \\
& \times \frac{\partial \alpha_{j}(r, z)}{\partial z}-\frac{K_{j}}{r}\left(1+\sin \left(2 \cdot \alpha_{j}(r, z)\right)\right)=0 \\
& \frac{\partial \sigma_{j}(r, z)}{\partial z}-2 K_{j} \cdot \sin \left(2 \cdot \alpha_{j}(r, z)\right) \\
& \times \frac{\partial \alpha_{j}(r, z)}{\partial r}+2 \cdot K_{j} \cdot \cos \left(2 \cdot \alpha_{j}(r, z)\right) \\
& \times \frac{\partial \alpha_{j}(r, z)}{\partial z}+\frac{K_{j}}{r} \cos \left(2 \cdot \alpha_{j}(r, z)\right)=0
\end{aligned}
$$

By transformation of Eqs. (4) we get the equation for a layer with number $j$ :

$$
\begin{aligned}
& \left\{\frac{\partial \sigma_{j}(r, z)}{\partial r}+\frac{\partial \sigma_{j}(r, z)}{\partial z} \tan (\beta)\right\} \\
& -2 \cdot K_{j} \cdot\left[\left\{\cos \left(2 \alpha_{j}(r, z)\right)+\sin \left(2 \alpha_{j}(r, z)\right) \cdot \tan (\beta)\right\} \frac{\partial \alpha_{j}(r, z)}{\partial r}\right. \\
& \left.+\left\{\sin \left(2 \alpha_{j}(r, z)\right)-\cos \left(2 \alpha_{j}(r, z)\right) \cdot \tan (\beta)\right\} \frac{\partial \alpha_{j}(r, z)}{\partial z}\right] \\
& +F_{j}(r, z)=0
\end{aligned}
$$

where

$$
\begin{aligned}
F(r, z)= & -\frac{K_{j}}{r}\left(1+\sin \left(2 \cdot \alpha_{j}(r, z)\right)\right) \\
& +\frac{K_{j}}{r} \cos \left(2 \cdot \alpha_{j}(r, z)\right) \cdot \tan (\beta)
\end{aligned}
$$

Let us consider the function $\alpha_{j}(r, z)$. It is defined on the contact surface by the boundary condition:

$\alpha_{j, j=\overline{1, \ldots, N}}(r, \tan (\beta) \cdot r+\Delta)=\left\{\begin{array}{l}\beta+\omega_{1}, r \in\left(a_{1}, a_{0}\right), \\ \beta+\omega_{j}, r \in\left(a_{j}, a_{j-1}\right.\end{array}\right]$

where $\omega_{j}=\frac{3 \pi}{4}+\psi_{j}, \quad j=\overline{1, \ldots, N} \quad$ (Figs. 1 and 2), $\psi_{j}(j=\overline{1, \ldots, N})$ is a constant angle which is defined by the direction of plastic shear in the contact area for layer with number $j$. It is known that $\alpha_{1}(r, 0)=\frac{\pi}{4}\left(r \in\left[a_{0}, \infty\right)\right)[1,2]$ (Fig. 2).

Taking into account Eq. (6) after some permutations of Eq. (5) we obtain the equation:

$\left\{\left.\frac{\partial \sigma_{j}(r, z)}{\partial r}\right|_{z=\tan (\beta) \cdot r+\Delta}+\left.\frac{\partial \sigma_{j}(r, z)}{\partial z}\right|_{z=\tan (\beta) \cdot r+\Delta} \cdot \tan (\beta)\right\}$

$-2 \cdot K_{j} \cdot\left[\{\cos (2 \cdot \beta)+\sin (2 \cdot \beta) \cdot \tan (\beta)\} \cdot \cos \left(2 \cdot \omega_{j}\right)\right.$

$\left.-\left\{\sin (2 \cdot \beta) \cdot(\tan (\beta))^{-1}-\cos (2 \cdot \beta)\right\} \cdot \tan (\beta) \cdot \sin \left(2 \cdot \omega_{j}\right)\right]$

$\times\left.\frac{\partial \alpha_{j}(r, z)}{\partial r}\right|_{z=\tan (\beta) \cdot r+\Delta}$

$+-2 \cdot K_{j} \cdot\left[\left\{\sin (2 \cdot \beta) \cdot(\tan (\beta))^{-1}-\cos (2 \cdot \beta)\right\}\right.$

$\times \tan (\beta) \cdot \cos \left(2 \cdot \omega_{j}\right)+\{\cos (2 \cdot \beta)+\sin (2 \cdot \beta) \cdot \tan (\beta)\}$

$\left.\times \sin \left(2 \cdot \omega_{j}\right)\right]\left.\cdot \frac{\partial \alpha_{j}(r, z)}{\partial z}\right|_{z=\tan (\beta) \cdot r+\Delta}+F_{j}(r, f(r))=0$

$r \in\left(a_{j}, a_{j-1}\right)$

Therefore,

$\left\{\left.\frac{\partial \sigma_{j}(r, z)}{\partial r}\right|_{z=\tan (\beta) \cdot r+\Delta}+\left.\frac{\partial \sigma_{j}(r, z)}{\partial z}\right|_{z=\tan (\beta) \cdot r+\Delta} \cdot \tan (\beta)\right\}$

$-2 \cdot K_{j} \cdot\left\{\cos \left(2 \cdot \omega_{j}\right)-\tan (\beta) \cdot \sin \left(2 \cdot \omega_{j}\right)\right\}$

$\times\left.\frac{\partial \alpha_{j}(r, z)}{\partial r}\right|_{z=\tan (\beta) \cdot r+\Delta}$

$+-2 \cdot K_{j} \cdot\left\{\tan (\beta) \cdot \cos \left(2 \cdot \omega_{j}\right)+\sin \left(2 \cdot \omega_{j}\right)\right\}$

$\times\left.\frac{\partial \alpha_{j}(r, z)}{\partial z}\right|_{z=\tan (\beta) \cdot r+\Delta}+F_{j}(r, f(r))=0, \quad r \in\left(a_{j}, a_{j-1}\right)$ 
A. Kravchuk, Z. Rymuza: Model of a superlattice indentation

Taking into account results of paper [3] we can get an additional equation:

$$
\begin{aligned}
& \left\{\left.\frac{\partial \alpha_{j}(r, z)}{\partial r}\right|_{z=\tan (\beta) \cdot r+\Delta} \cdot(-\tan (\beta))+\left.\frac{\partial \alpha_{j}(r, z)}{\partial z}\right|_{z=\tan (\beta) \cdot r+\Delta}\right\} \\
= & -\operatorname{ctg}\left(\omega_{j}\right)\left\{\left.\frac{\partial \alpha_{j}(r, z)}{\partial r}\right|_{z=\tan (\beta) \cdot r+\Delta}+\left.\frac{\partial \alpha_{j}(r, z)}{\partial z}\right|_{z=\tan (\beta) \cdot r+\Delta v} \tan (\beta)\right\}
\end{aligned}
$$

Using Eqs. (7) and (8), we obtain that the function $s$ satisfy the differential equation at the bound of the layer with number $j$ :

$\frac{\mathrm{d} \sigma_{j}}{\mathrm{~d} r}+2 K_{j} \cdot \frac{\mathrm{d} \alpha_{j}}{\mathrm{~d} r}=-F_{j}(r, \tan (\beta) \cdot r+\Delta), r \in\left(a_{j}, a_{j-1}\right)$

From Eq. (9) we obtain the solution in the form:

$$
\begin{aligned}
& \sigma_{j}(r, \tan (\beta) \cdot r+\Delta)=-2 \cdot K_{j} \cdot \alpha_{j}(r, \tan (\beta) \cdot r+\Delta) \\
& +\left(\sigma_{j}\left(a_{j-1}, h_{j-1}\right)+2 \cdot K_{j} \cdot \alpha_{j}\left(a_{j-1}, h_{j-1}\right)\right) \\
& -\int_{a_{j-1}}^{r} F_{j}(r, \tan (\beta) \cdot r+\Delta) \mathrm{d} r, \quad r \in\left(a_{j}, a_{j-1}\right]
\end{aligned}
$$

where $\quad h_{j}=\tan (\beta) \cdot a_{j}+\Delta \quad$ (Fig. 2), $\quad \alpha_{1}(a, 0)=\frac{\pi}{4}$, $\alpha_{j}\left(a_{j-1}, h_{j-1}\right)=\beta+\frac{3 \cdot \pi}{4}+\psi_{j}$. It is necessary to emphasize that the constants $\sigma_{j}\left(a_{j-1}, h_{j-1}\right)$ have to be determined from the boundary conditions on the flat boundary of each layer.

\section{Successive determination of the constants; Solution of the problem}

Let us consider the first layer $(j=1)$. It is known $[1,2]$ that $\sigma_{z, 1}(r, 0)=0$ on the free boundary of a multilayer body $(z=0, r \in[a, \infty)$, (Figs. 1 and 2)). It means that

$\sigma_{1}\left(a_{0}, h_{0}\right)=K_{1}, \quad r \in[a, \infty)$

Therefore, we obtain from Eqs. (10) and (11),

$$
\begin{aligned}
& \sigma_{1}(r, \tan (\beta) \cdot r+\Delta)=-2 \cdot K_{1} \cdot\left(\beta+\frac{\pi}{2}+\psi_{1}+\frac{1}{2}\right) \\
& +K_{1}\left(1-\cos \left(2 \cdot \beta+2 \cdot \psi_{1}\right)\right. \\
& \left.-\sin \left(2 \cdot \beta+2 \cdot \psi_{1}\right) \cdot \tan (\beta)\right) \ln \left(\frac{r}{a}\right), \quad r \in\left(a_{1}, a_{0}\right)
\end{aligned}
$$

Further an equation is valid successively for $j=2, N$ :

$\sigma_{z, j}\left(r, h_{j-1}\right)=\sigma_{z, j-1}\left(r, h_{j-1}\right), \quad r \in\left[a_{j-1}, \infty\right)$

Hence $\sigma_{z, j}\left(a_{j-1}, h_{j-1}\right)=\sigma_{z, j-1}\left(a_{j-1}, h_{j-1}\right)$. Finally, taking into account Eq. (2), we get:

$\sigma_{j}\left(a_{j-1}, h_{j-1}\right)=\sigma_{j-1}\left(a_{j-1}, h_{j-1}\right)$

$-K_{j-1} \cdot \cos \left(2 \cdot \beta+2 \cdot \psi_{j-1}\right)+K_{j} \cdot \cos \left(2 \cdot \beta+2 \cdot \psi_{j}\right)$

Int. J. Mat. Res. (formerly Z. Metallkd.) 98 (2007) 5
Thus:

$$
\begin{aligned}
\sigma_{j}= & \sigma_{j}\left(a_{j-1}, h_{j-1}\right)+K_{j}\left(1-\cos \left(2 \cdot \beta+2 \cdot \psi_{j}\right)\right. \\
& \left.-\sin \left(2 \cdot \beta+2 \cdot \psi_{j}\right) \cdot \tan (\beta)\right) \ln \left(\frac{r}{a_{j-1}}\right)
\end{aligned}
$$

$r \in\left(a_{j}, a_{j-1}\right]$

Taking into account Eqs. (12) and (13) we can consider a function $\sigma_{z}, r \in[0, a)$ :

$\sigma_{z}=\left\{\begin{array}{l}\sigma_{1}-K_{1} \cdot \cos \left(2 \cdot \beta+2 \cdot \psi_{1}\right), r \in\left(a_{1}, a\right) \\ \cdots \\ \sigma_{j}-K_{j} \cdot \cos \left(2 \cdot \beta+2 \cdot \psi_{j}\right), r \in\left(a_{j}, a_{j-1}\right] \\ \cdots \\ \sigma_{N}-K_{N} \cdot \cos \left(2 \cdot \beta+2 \cdot \psi_{N}\right), r \in\left(a_{N}, a_{N-1}\right]\end{array}\right.$

The applied force $F$ is determined by the equation [1]:

$$
\begin{aligned}
F & =2 \pi \int_{0}^{a}\left(-\sigma_{z}\right) \cdot r \mathrm{~d} r \\
& =-2 \pi \sum_{j=1}^{N} \int_{a_{j}}^{a_{j-1}}\left(\sigma_{j}-K_{j} \cdot \cos \left(2 \cdot \beta+2 \cdot \psi_{j}\right)\right) \cdot r \mathrm{~d} r
\end{aligned}
$$

Taking into account Eq. (15), the Meyers hardness (HM) is defined by the following equation:

$\mathrm{HM}=\frac{F}{\pi \cdot a^{2}}=\frac{1}{\pi \cdot a^{2}}\left(2 \pi \int_{0}^{a}\left(-\sigma_{z}\right) \cdot r \mathrm{~d} r\right)$

\section{Results of calculations}

The cone angle, the yield stress of the first layer, and the direction of the plastic shear of each layer in the area of contact with the conical indenter significantly influence the distribution of $\sigma_{z}(r)$ [Eqs. (14)] (Figs. 3-5). On the con-

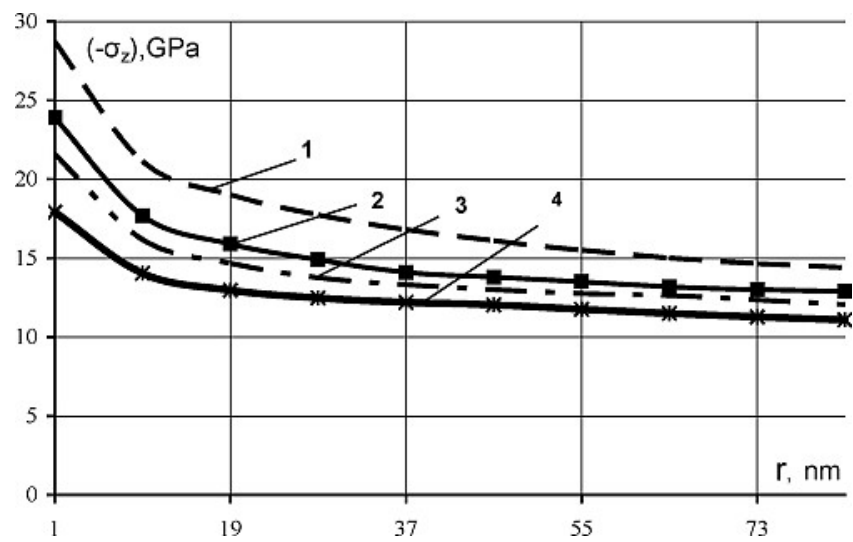

Fig. 3. Distribution of the stress $\sigma_{z}(r)$ [Eq. (14)] for different angles $\beta$ (Fig. 1). Number of layers $N=5$; maximal depth of penetration $\Delta=44.59 \mathrm{~nm}$; set of layer thickness $d_{1}=2 \mathrm{~nm}, d_{2}=20 \mathrm{~nm}$, $d_{3}=5 \mathrm{~nm}, d_{4}=10 \mathrm{~nm}, d_{5}=12 \mathrm{~nm}$; yield stress for each layer $\sigma_{s, j(j=\overline{1, \ldots, 5})}=2 \cdot K_{j}, K_{1}=2 \mathrm{GPa}, K_{2}=4 \mathrm{GPa}, K_{3}=1.5 \mathrm{GPa}, K_{4}=$ $2.5 \mathrm{GPa}, K_{5}=4 \mathrm{GPa}, \psi_{j(j=\overline{1, \ldots, 5})}=\pi / 4.1: \beta=-\pi / 18 ; 2: \beta=-\pi / 10$; 3: $\beta=-\pi / 8 ; 4: \beta=-\pi / 6$. 
A. Kravchuk, Z. Rymuza: Model of a superlattice indentation

trary, the distribution of $\sigma_{z}(r)$ in the area of contact does not depend on the thickness of the layers (Fig. 6).

The measured hardness of a multilayer system increases with decreasing angle $\beta$ (Fig. 1) of the indenter (Fig. 7) or increasing yield stress of the first layer (Fig. 8). The hardness significantly depends on direction of plastic shear of each layer in the area of contact (Fig. 9), but does not depend on the thickness of a layer (Fig. 10).

The $F-\Delta$ (force-penetration depth) diagram has been determined in some cases of superlattice coatings (Fig. 11). It was established that the plastic properties of each layer cannot separately be defined by this diagram in the case of application of a conical indenter.

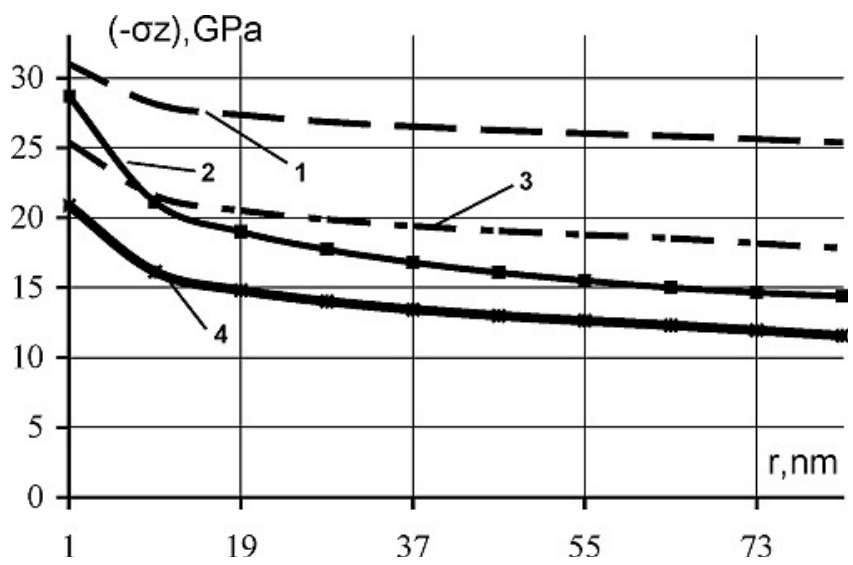

Fig. 4. Distribution of the stress $\sigma_{z}(r)$ [Eq. (14)] for different sets of the plastic properties $K_{j} . \beta=-\pi / 18$; number of layers $N=5$; maximal depth of penetration $\Delta=44.59 \mathrm{~nm}$; set of layer thicknesses $d_{1}=2 \mathrm{~nm}, d_{2}=20 \mathrm{~nm}, d_{3}=5 \mathrm{~nm}, d_{4}=10 \mathrm{~nm}, d_{5}=12 \mathrm{~nm}$; yield stress for each layer $\sigma_{s, j(j=\overline{1, \ldots, 5})}=2 \cdot K_{j} ; \psi_{j(j=\overline{1, \ldots, 5})}=\pi / 4$.

$1: K_{1}=4 \mathrm{GPa}, K_{2}=2 \mathrm{GPa}, K_{3}=4 \mathrm{GPa}, K_{4}=2.5 \mathrm{GPa}, K_{5}=1.5 \mathrm{GPa}$;

2: $K_{1}=2 \mathrm{GPa}, K_{2}=4 \mathrm{GPa}, K_{3}=1.5 \mathrm{GPa}, K_{4}=2.5 \mathrm{GPa}, K_{5}=4 \mathrm{GPa}$;

3: $K_{1}=2.5 \mathrm{GPa}, K_{2}=4 \mathrm{GPa}, K_{3}=1.5 \mathrm{GPa}, K_{4}=4 \mathrm{GPa}, K_{5}=2 \mathrm{GPa}$;

4: $K_{1}=1.5 \mathrm{GPa}, K_{2}=2 \mathrm{GPa}, K_{3}=4 \mathrm{GPa}, K_{4}=4 \mathrm{GPa}, K_{5}=2.5 \mathrm{GPa}$.

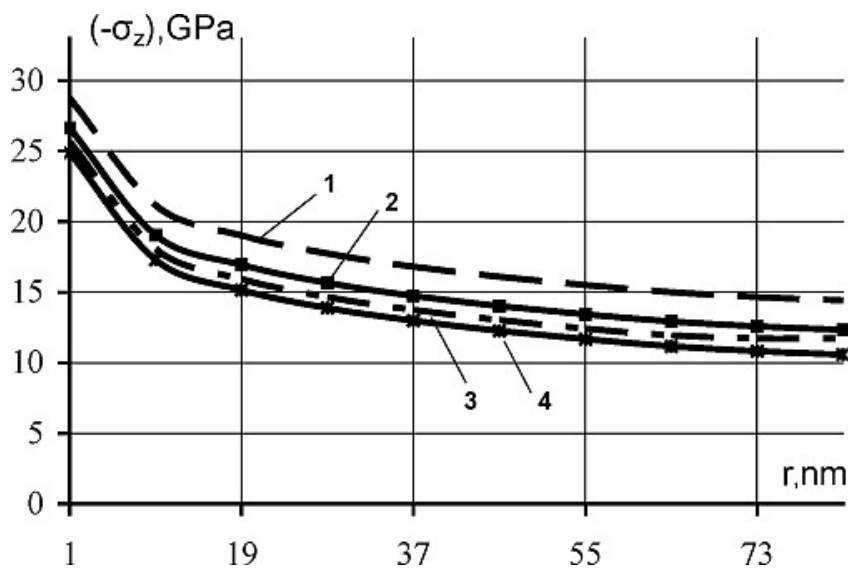

Fig. 5. Distribution of the stress $\sigma_{z}(r)$ [Eq. (14)] for different sets of the direction of the plastic shear $\psi_{j}$ in the area of contact. $\beta=-\pi / 18$; number of layers $N=5$; maximal depth of penetration $\Delta=$ $44.59 \mathrm{~nm}$; set of layer thicknesses $d_{1}=2 \mathrm{~nm}, d_{2}=20 \mathrm{~nm}, d_{3}=$ $5 \mathrm{~nm}, d_{4}=10 \mathrm{~nm}, d_{5}=12 \mathrm{~nm}$; yield stress for each layer $\sigma_{s, j(j=\overline{1, \ldots, 5})}=2 \cdot K_{j} ; K_{1}=2 \mathrm{GPa}, K_{2}=4 \mathrm{GPa}, K_{3}=1.5 \mathrm{GPa}, K_{4}=$ $2.5 \mathrm{GPa}, K_{5}=4 \mathrm{GPa}$.

$1: \psi_{j(j=\overline{1, \ldots .5})}=\pi / 4$

$2: \psi_{1}=\pi / 4, \psi_{2}=0, \psi_{3}=0, \psi_{4}=\pi / 4, \psi_{5}=\pi / 4$

$3: \psi_{1}=\pi / 4, \psi_{2}=0, \psi_{3}=\pi / 4, \psi_{4}=0, \psi_{5}=\pi / 4$;

$4: \psi_{1}=0, \psi_{2}=0, \psi_{3}=\pi / 4, \psi_{4}=\pi / 4, \psi_{5}=\pi / 4$.

\section{Conclusions}

A model of superlattice (multilayer) indentation by a rigid conical indenter has been created. The penetration of the indenter has been studied on the basis of the Haar and von Karman hypothesis. The analytical distribution of the contact stress has been obtained.

The yield stress of the first layer and the direction of the plastic shear of each layer in the area of contact with the conical indenter significantly influence the hardness of the whole superlattice system. The hardness of a multilayer system increases with increasing yield stress of the first layer.

The force-penetration depth diagram has been determined in some cases of superlattice coatings. It was established that the plastic properties of each layer cannot separately be defined by this diagram in the case of application of a conical indenter.

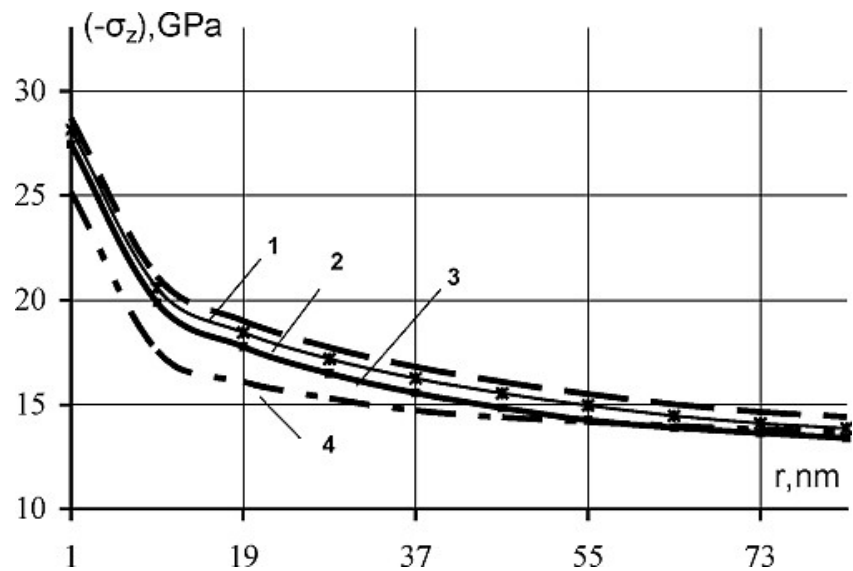

Fig. 6. Distribution of the stress $\sigma_{z}(r)$ [Eq. (14)] for different sets of layer depth $d_{j} . \beta=\pi /-18$; number of layers $N=5$; maximal depth of penetration $\Delta=44.59 \mathrm{~nm}$; yield stress for each layer $\sigma_{s, j(j=\overline{1, \ldots, 5})}=$ $2 \cdot K_{j} ; K_{1}=2 \mathrm{GPa}, K_{2}=4 \mathrm{GPa}, K_{3}=1.5 \mathrm{GPa}, K_{4}=2.5 \mathrm{GPa}, K_{5}=$ $4 \mathrm{GPa} ; \psi_{j(j=1, \ldots, 5)}=\pi / 4$.

1: $d_{1}=2 \mathrm{~nm}, d_{2}=20 \mathrm{~nm}, d_{3}=5 \mathrm{~nm}, d_{4}=10 \mathrm{~nm}, d_{5}=12 \mathrm{~nm}$

2: $d_{1}=20 \mathrm{~nm}, d_{2}=5 \mathrm{~nm}, d_{3}=2 \mathrm{~nm}, d_{4}=10 \mathrm{~nm}, d_{5}=12 \mathrm{~nm}$;

3: $d_{1}=5 \mathrm{~nm}, d_{2}=2 \mathrm{~nm}, d_{3}=20 \mathrm{~nm}, d_{4}=12 \mathrm{~nm}, d_{5}=10 \mathrm{~nm}$;

4: $d_{1}=12 \mathrm{~nm}, d_{2}=10 \mathrm{~nm}, d_{3}=20 \mathrm{~nm}, d_{4}=5 \mathrm{~nm}, d_{5}=2 \mathrm{~nm}$.

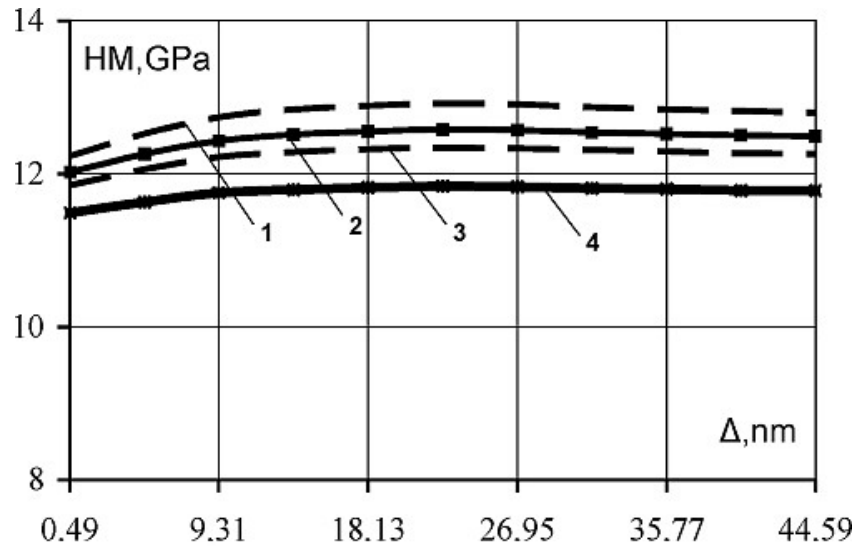

Fig. 7. Variation of the hardness [Eq. (16)] for different angles $\beta$ of the conical indenter. Number of layers $N=5$; maximal depth of penetration $\Delta=44.59 \mathrm{~nm}$; set of layer thicknesses $d_{1}=2 \mathrm{~nm}, d_{2}=20 \mathrm{~nm}$, $d_{3}=5 \mathrm{~nm}, d_{4}=10 \mathrm{~nm}, d_{5}=12 \mathrm{~nm}$; yield stress for each layer $\sigma_{s, j(j=\overline{1, \ldots, 5})}=2 \cdot K_{j}, K_{1}=2 \mathrm{GPa}, K_{2}=4 \mathrm{GPa}, K_{3}=1.5 \mathrm{GPa}, K_{4}=$ $2.5 \mathrm{GPa}, K_{5}=4 \mathrm{GPa}, \psi_{j(j=\overline{1, \ldots, 5})}=\pi / 4$.

$1: \beta=-\pi / 18 ; 2: \beta=-\pi / 10 ; 3: \beta=-\pi / 8 ; 4: \beta=-\pi / 6$.

Int. J. Mat. Res. (formerly Z. Metallkd.) 98 (2007) 5 
A. Kravchuk, Z. Rymuza: Model of a superlattice indentation

HM, GPa

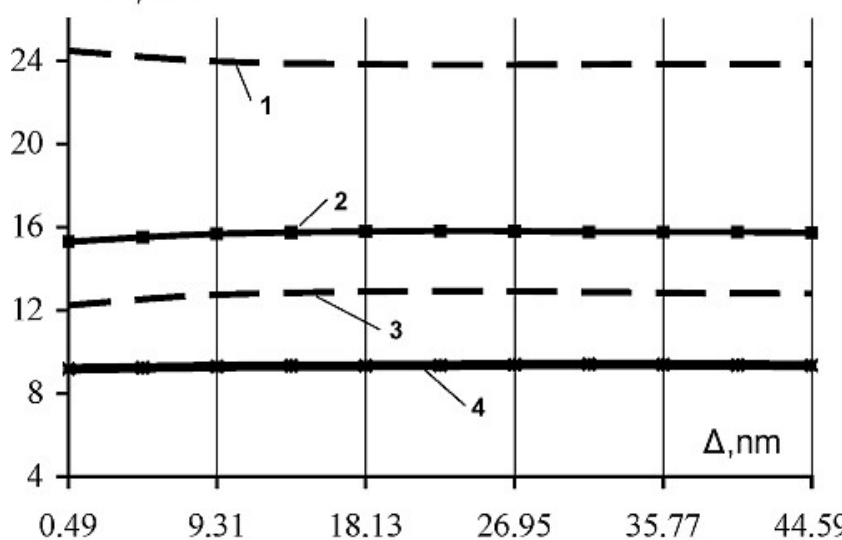

Fig. 8. Variation of the hardness [Eq. (16)] for different sets of the plastic properties $K_{j} . \beta=-\pi / 18$; number of layers $N=5$; maximal depth of penetration $\Delta=44.59 \mathrm{~nm}$; set of layer thicknesses $d_{1}=$ $2 \mathrm{~nm}, d_{2}=20 \mathrm{~nm}, d_{3}=5 \mathrm{~nm}, d_{4}=10 \mathrm{~nm}, d_{5}=12 \mathrm{~nm}$; yield stress for each layer $\sigma_{s, j(j=\overline{1, \ldots .5})}=2 \cdot K_{j} ; \psi_{j(j=\overline{1 . \ldots .5})}=\pi / 4$.

1: $K_{1}=4 \mathrm{GPa}, K_{2}=2 \mathrm{GPa}, K_{3}=4 \mathrm{GPa}, K_{4}=2.5 \mathrm{GPa}, K_{5}=1.5 \mathrm{GPa}$; 2: $K_{1}=2.5 \mathrm{GPa}, K_{2}=4 \mathrm{GPa}, K_{3}=1.5 \mathrm{GPa}, K_{4}=4 \mathrm{GPa}, K_{5}=2 \mathrm{GPa}$; 3: $K_{1}=2 \mathrm{GPa}, K_{2}=4 \mathrm{GPa}, K_{3}=1.5 \mathrm{GPa}, K_{4}=2.5 \mathrm{GPa}, K_{5}=4 \mathrm{GPa}$ 4: $K_{1}=1.5 \mathrm{GPa}, K_{2}=2 \mathrm{GPa}, K_{3}=4 \mathrm{GPa}, K_{4}=4 \mathrm{GPa}, K_{5}=2.5 \mathrm{GPa}$.

$\mathrm{HM}, \mathrm{GPa}$

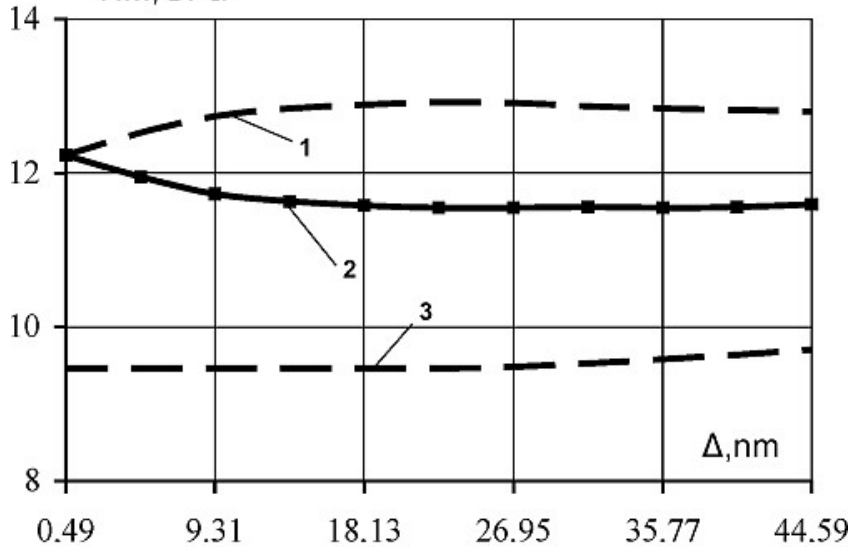

Fig. 9. Variation of the hardness [Eq. (16)] for different sets of the direction of plastic shear $\psi_{j}$ in the area of contact. $\beta=-\pi / 18$; number of layers $N=5$; maximal depth of penetration $\Delta=44.59 \mathrm{~nm}$; set of layer thicknesses $d_{1}=2 \mathrm{~nm}, d_{2}=20 \mathrm{~nm}, d_{3}=5 \mathrm{~nm}, d_{4}=10 \mathrm{~nm}$, $d_{5}=12 \mathrm{~nm} ;$ yield stress for each layer $\sigma_{s, j(j=\overline{1, \ldots 5})}=2 \cdot K_{j}$; $K_{1}=2 \mathrm{GPa}, K_{2}=4 \mathrm{GPa}, K_{3}=1.5 \mathrm{GPa}, K_{4}=2.5 \mathrm{GPa}, K_{5}=4 \mathrm{GPa}$.

$1: \psi_{j(j=\overline{1, \ldots .5})}=\pi / 4$

$2: \psi_{1}=\pi / 4, \psi_{2}=0, \psi_{3}=\pi / 4, \psi_{4}=0, \psi_{5}=\pi / 4$

$3: \psi_{1}=0, \psi_{2}=0, \psi_{3}=\pi / 4, \psi_{4}=\pi / 4, \psi_{5}=\pi / 4$.

A. Kravchuk acknowledges the support from the Kasa Mianowskiego Fund (Warsaw, Poland). The work was performed in the frame of the internal research programme of the Institute of Micromechanics and Photonics of the Warsaw University of Technology.

\section{References}

[1] A.J. Ishlinsky, D.D. Ivlev: Mathematical Theory of Plasticity, Fizmatlit, Moskow (2001) [in Russian].

[2] R.T. Shield: Proc. Roy. Soc. Series A 233 (1955) 267.

[3] A. Kravchuk, R. Buzio, U. Valbusa, Z. Rymuza: Technische Mechanik, Band 25, Heft 2 (2005) 81.

(Received October 11, 2006; accepted January 14, 2007)

\section{$\mathrm{HM}, \mathrm{GPa}$}

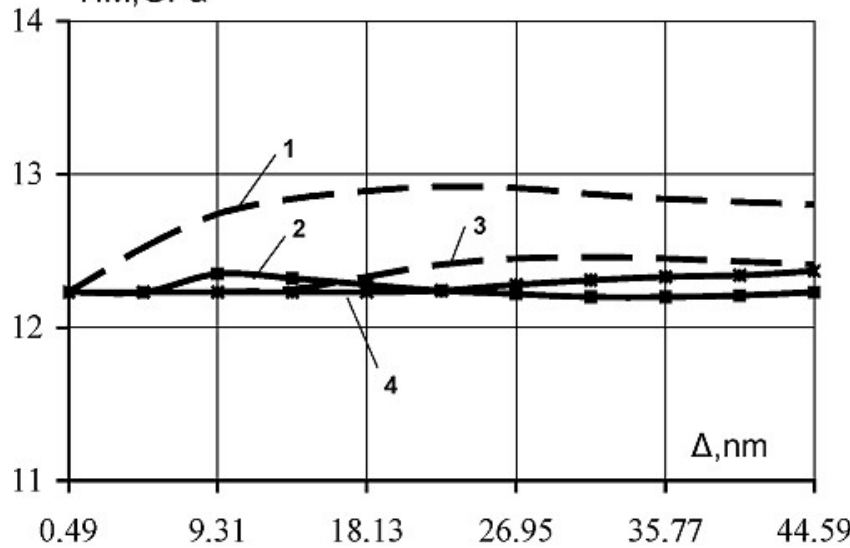

Fig. 10. Variation of the hardness [Eq. (16)] for different sets of layer thicknesses $d_{j} . \beta=-\pi / 18$; number of layers $N=5$; maximal depth of penetration $\Delta=44.59 \mathrm{~nm}$; set of layer thicknesses $d_{1}=2 \mathrm{~nm}$, $d_{2}=20 \mathrm{~nm}, d_{3}=5 \mathrm{~nm}, d_{4}=10 \mathrm{~nm}, d_{5}=12 \mathrm{~nm}$; yield stress for each layer $\sigma_{s, j(j=\overline{1 . \ldots .5})}=2 \cdot K_{j} ; K_{1}=2 \mathrm{GPa}, K_{2}=4 \mathrm{GPa}, K_{3}=1.5 \mathrm{GPa}$, $K_{4}=2.5 \mathrm{GPa}, K_{5}=4 \mathrm{GPa} ; \psi_{j(j=\overline{1, \ldots .5})}=\pi / 4$.

$1: d_{1}=2 \mathrm{~nm}, d_{2}=20 \mathrm{~nm}, d_{3} \stackrel{j(j=1, \ldots, 5)}{=}, d_{4}=10 \mathrm{~nm}, d_{5}=12 \mathrm{~nm}$

2: $d_{1}=5 \mathrm{~nm}, d_{2}=2 \mathrm{~nm}, d_{3}=20 \mathrm{~nm}, d_{4}=12 \mathrm{~nm}, d_{5}=10 \mathrm{~nm}$

3: $d_{1}=12 \mathrm{~nm}, d_{2}=10 \mathrm{~nm}, d_{3}=20 \mathrm{~nm}, d_{4}=5 \mathrm{~nm}, d_{5}=2 \mathrm{~nm}$;

4: $d_{1}=20 \mathrm{~nm}, d_{2}=5 \mathrm{~nm}, d_{3}=2 \mathrm{~nm}, d_{4}=10 \mathrm{~nm}, d_{5}=12 \mathrm{~nm}$.

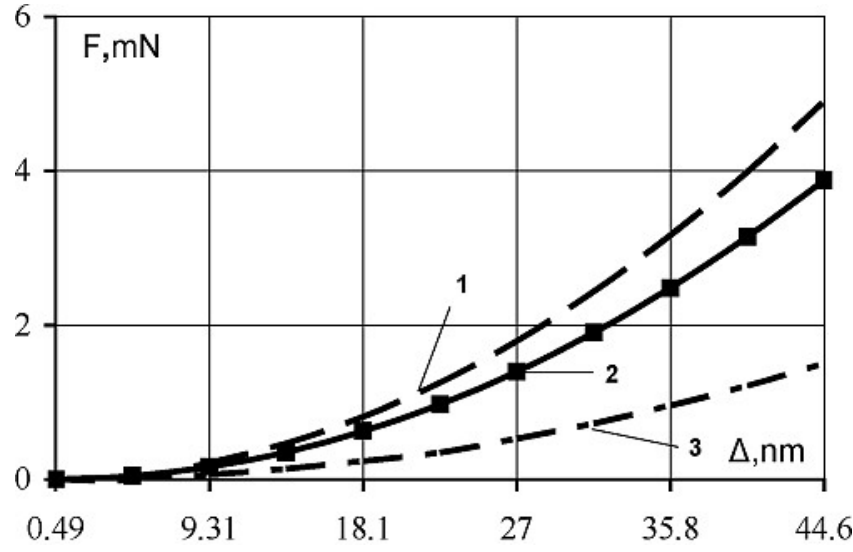

Fig. 11. Force-penetration depth diagram. $\beta=-\pi / 18 ; \beta=-\pi / 18$; number of layers $N=5$; maximal depth of penetration $\Delta=$ $44.59 \mathrm{~nm} ; \sigma_{s, j(j=\overline{1, \ldots .5})}=2 \cdot K_{j}$.

1: $d_{1}=20 \mathrm{~nm}, d_{2}=12 \mathrm{~nm}, d_{3}=10 \mathrm{~nm}, d_{4}=5 \mathrm{~nm}, d_{5}=2 \mathrm{~nm}$ $K_{1}=4 \mathrm{GPa}, K_{2}=4 \mathrm{GPa}, K_{3}=2.5 \mathrm{GPa}, K_{4}=2 \mathrm{GPa}, K_{5}=1.5 \mathrm{GPa}$; $\psi_{j(j=1,5,5)}=\pi / 4$;

2: $d_{1}=20 \mathrm{~nm}, d_{2}=12 \mathrm{~nm}, d_{3}=10 \mathrm{~nm}, d_{4}=5 \mathrm{~nm}, K_{1}=4 \mathrm{GPa}$ $K_{2}=4 \mathrm{GPa}, K_{3}=2.5 \mathrm{GPa}, K_{4}=2 \mathrm{GPa}, K_{5}=1.5 \mathrm{GPa} ; \quad \psi_{1}=0$, $\psi_{2}=\pi / 4, \psi_{3}=0, \psi_{4}=\pi / 4, \psi_{5}=\pi / 4$

3: $d_{1}=20 \mathrm{~nm}, d_{2}=12 \mathrm{~nm}, d_{3}=10 \mathrm{~nm}, d_{4}=5 \mathrm{~nm}, K_{1}=1.5 \mathrm{GPa}$ $K_{2}=4 \mathrm{GPa}, \quad K_{3}=2.5 \mathrm{GPa}, \quad K_{4}=2 \mathrm{GPa}, \quad K_{5}=4 \mathrm{GPa} ; \quad \psi_{1}=0$, $\psi_{2}=\pi / 4, \psi_{3}=0, \psi_{4}=\pi / 4, \psi_{5}=\pi / 4$.

\section{Correspondence address}

Prof. Zygmunt Rymuza

Warsaw University of Technology

Institute of Micromechanics and Photonics

ul. Sw. A. Boboli 8, 02-525 Warszawa, Poland

Tel.: +48226608540

Fax: +48226608601

E-mail: z.rymuza@mchtr.pw.edu.pl

You will find the article and additional material by entering the document number MK101481 on our website at www.ijmr.de 